\title{
Changes in normal polymorphonuclear leucocyte motility after ingestion of IgG aggregates
}

\author{
D. H. GODDARD, A. P. KIRK, K. A. B ROWN, D. MCCARTHY, \\ G. D. JOHNSON, ANDE.J. HOLBOROW
}

From the M.R.C. Immunology Section, Bone and Joint Research Unit, The London Hospital Medical College, Turner Street, London E1, and the *Department of Plant Virology, Queen Mary College, Mile End Road, London E1

SUMMARY Changes in normal polymorphonuclear leucocyte (PMN) motility after membranebinding and internalisation of $\mathrm{IgG}$ aggregates (a model of soluble immune complexes) have been studied by the micropore filter assay. The results have confirmed that IgG aggregates stimulate as well as inhibit PMN chemotaxis. These effects are dependent on the size and concentration of the IgG aggregates in solution as well as the length of time of incubation. Stimulated chemotaxis was observed in a small subset of the whole PMN population which was apparent only when cell distribution through the filters was analysed. These results indicate the need for caution when drawing conclusions about PMN function from results obtained by these assay techniques.

Membrane binding and internalisation of soluble immune complexes by polymorphonuclear leucocytes have been shown to result in altered function as measured by in-vitro tests-for example, cell surface charge. ${ }^{1}$ Despite the high levels of circulating soluble immune complexes often found in rheumatoid arthritis (RA) a consistent defect in PMN chemotaxis has not been found, although in the preceding paper we have reported reduced chemotaxis in RA blood PMNs, and have identified immunoglobulin inclusions in the cytoplasm of these cells, thought to be phagocytosed immune complexes. These results are in agreement with earlier findings of Mowat and Baum. $^{2}$

In this study we have used stable IgG aggregates with biological activity resembling soluble immune complexes to study the changes that occur in normal PMN motility after their uptake by these cells, using the micropore filter assay. We have measured effects on migration of PMNs through the filters by both the 'leading front technique' ${ }^{3}$ and by analysing cell distributions through the filters. Double fluorochrome immunofluorescence staining was used to detect and differentiate between internalised and bound immunoglobulin.

Accepted for publication 8 June 1983

Correspondence to Dr D. H. Goddard, Department of Rheumatology, Westminster Hospital, Horseferry Road, London SW1P 2AP.

\section{Materials and methods}

Polymorphonuclear leucocytes were isolated from the peripheral blood of normal volunteers by the methods described in the preceding paper. ${ }^{4}$

Stable IgG aggregates were prepared according to the method of McCarthy et al. ${ }^{5}$ Aggregates prepared in this way remain stable when separated into different sizes by sucrose-density gradient centrifugation.

Cell motility was measured by the micropore filter assay. Details of this assay and the method of immunofluorescent staining of PMNs have been described in detail in the preceding paper. ${ }^{4}$

PMNs were incubated with IgG aggregates of different sizes and concentrations for different lengths of time in a water-bath at $37^{\circ} \mathrm{C}$ before measuring cell motility. Details of these experiments are given in the results section.

\section{Results}

KINETICS

PMNs were incubated with IgG aggregates $\left(2 \times 10^{6}\right.$ PMNs: $200 \mu \mathrm{g}$ unfractionated IgG aggregate) at $37^{\circ} \mathrm{C}$ in a water-bath. Maximal uptake of aggregate occurred between 30 and 60 minutes' incubation (Fig. 1). Immunofluorescent staining of IgG inclusions had largely disappeared after incubation for 360 minutes at $37^{\circ} \mathrm{C}$. PMN viability was reassessed 
after 360 minutes' incubation by measuring the ability of cells to exclude trypan blue dye; this showed that $98 \%$ remained viable. PMNs were rechallenged with fresh IgG aggregates after 330 minutes' incubation and incubated for a further 30 minutes at $37^{\circ} \mathrm{C}$. Fig. 1 shows that these cells developed further immunoglobulin inclusions, indicating that further phagocytosis of IgG aggregates had occurred.

A significant reduction in PMN chemotaxis but not random migration occurred after 30 minutes' incubation with IgG aggregates ( $\mathrm{p}<0 \cdot 05$, paired $t$ test) (Fig. 2). After 60 minutes' and 180 minutes' incubation, chemotaxis and random motility had increased above the control value. However, after 360 minutes' incu-

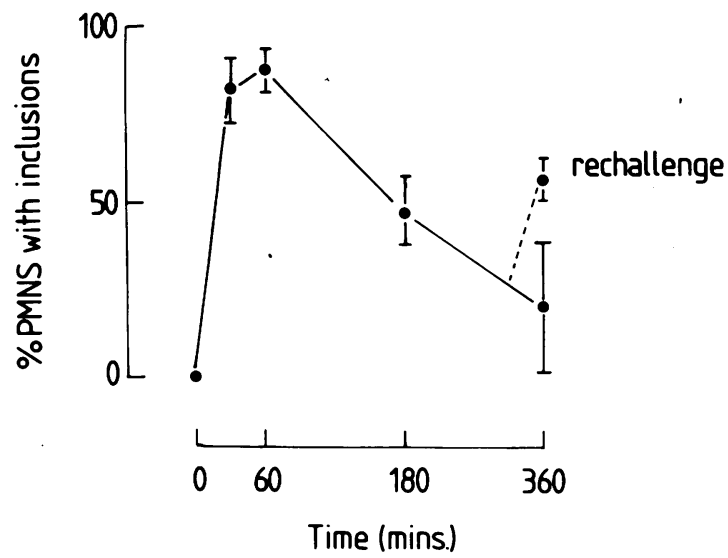

Fig. 1 Kinetics: Immunofluorescence showed maximal uptake of IgG aggregates occurred between 30 and 60 minutes' incubation. Further uptake of aggregates occurred after rechallenge at 360 minutes' incubation (dotted line). bation only chemotaxis remained increased above the control value. When PMNs were rechallenged with fresh IgG aggregates, and incubated for a further 30 minutes, a further significant fall in chemotaxis occurred ( $<<0.01$, paired $t$ test) (Fig. 2).

When the filters were analysed in a step-wise fashion and the distribution of migrating cells was measured, it appeared that the observed increase in chemotaxis and random motility was due to a small subset of PMNs that had migrated further and faster through the filters, while the main population remained distributed within the first $45-60 \mu \mathrm{m}$ of the filters (Table 1).

EFFECTS OF IGG AGGREGATE CONCENTRATION PMNs were incubated with doubling dilutions of IgG aggregates for 30 minutes at $37^{\circ} \mathrm{C}$ in a water-bath.

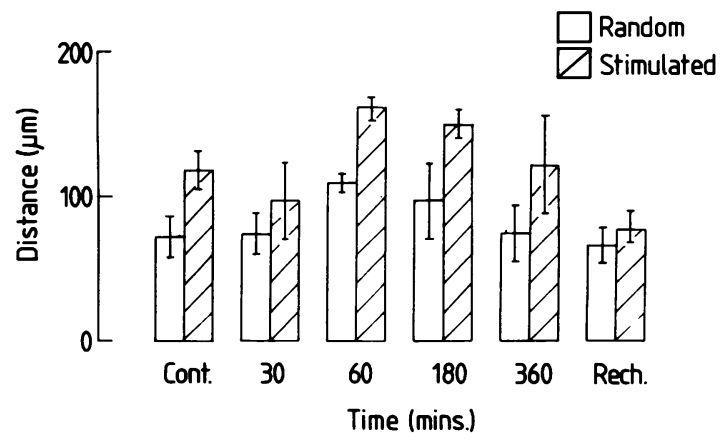

Fig. 2 Kinetics: Chemotaxis but not random migration was reduced after 30 minutes' incubation. Stimulation of chemotaxis and random migration occurred a fter 60 and 180 minutes' incubation. Chemotaxis was reduced after rechallenge with IgG aggregates.

Table 1 Migration of PMNs after incubation with un fractionated IgG aggregates: figures shown are the mean percentages of PMNs at different depths through the filters

\begin{tabular}{|c|c|c|c|c|c|c|c|c|c|c|c|c|c|}
\hline \multirow{2}{*}{$\begin{array}{l}\text { Duration of preincubation } \\
\text { with IgG aggregates (min) }\end{array}$} & & \multicolumn{12}{|c|}{ Filter depth } \\
\hline & & 30 & 45 & 60 & 75 & 90 & 105 & 120 & 135 & 150 & 165 & 180 & 195 \\
\hline \multicolumn{14}{|l|}{ Stimulated migration } \\
\hline 0 (control: no aggr.) & & 71 & 16 & 8 & 3 & 1 & $0 \cdot 4$ & $0 \cdot 3$ & $0 \cdot 1$ & $0 \cdot 1$ & & & \\
\hline 30 & & 90 & 8 & 2 & $0 \cdot 8$ & $0 \cdot 3$ & $0 \cdot 2$ & $0 \cdot 1$ & $0 \cdot 1$ & & & & \\
\hline 60 & & 82 & 6 & 3 & $1 \cdot 4$ & $1 \cdot 5$ & $1 \cdot 4$ & $1 \cdot 2$ & $1 \cdot 0$ & $0 \cdot 7$ & $0 \cdot 7$ & $0 \cdot 4$ & $0 \cdot 2$ \\
\hline 360 & & 88 & 5 & 3 & $1 \cdot 4$ & $1 \cdot 1$ & 0.5 & 0.6 & 0.5 & $0 \cdot 3$ & $0 \cdot 1$ & $0 \cdot 1$ & \\
\hline 360 (rechallenge) & & 94 & 4 & $1 \cdot 2$ & $0 \cdot 4$ & $0 \cdot 2$ & & & & & & & \\
\hline \multicolumn{14}{|l|}{ Random migration } \\
\hline 0 (control: no aggr.) & & 82 & 13 & 5 & $0 \cdot 8$ & $0 \cdot 2$ & $0 \cdot 1$ & & & & & & \\
\hline 30 & & 92 & 6 & 1 & 0.4 & $0 \cdot 2$ & $0 \cdot 1$ & $0 \cdot 1$ & & & & & \\
\hline 60 & - & 91 & 5 & 2 & $1 \cdot 2$ & 0.6 & $0 \cdot 3$ & $0 \cdot 1$ & & & & & \\
\hline 180 & & 91 & 5 & $2 \cdot 4$ & 0.8 & 0.2 & $0 \cdot 1$ & & & & & & \\
\hline 360 (rechallenge) & & 98 & 3 & 0.4 & $0 \cdot 1$ & & & & & & & & \\
\hline
\end{tabular}


Over the concentration range $25-200 \mu \mathrm{g} / \mathrm{ml}$ of aggregates the percentage of PMNs developing inclusions was directly proportional to $\log _{10}$ of the protein concentration (Fig. 3). At concentrations of 200 $\mu \mathrm{g} / \mathrm{ml}$ chemotaxis but not random migration was significantly reduced ( $p<0.01$, paired $t$ test) (Fig. 4). However, at a concentration of $50 \mu \mathrm{g} / \mathrm{ml}$ and less no alteration in chemotaxis or random migration occurred.

\section{EFFECTS OF IGG AGGREGATE SIZE}

In preliminary experiments PMNs were incubated with aggregates of different sizes for 30 minutes at $37^{\circ} \mathrm{C}$ in a water-bath. These aggregate fractions had the following mean sedimentation coefficients: fraction $3(<20 S)$, fraction $6(40 S)$, fraction $9(90 S)$,

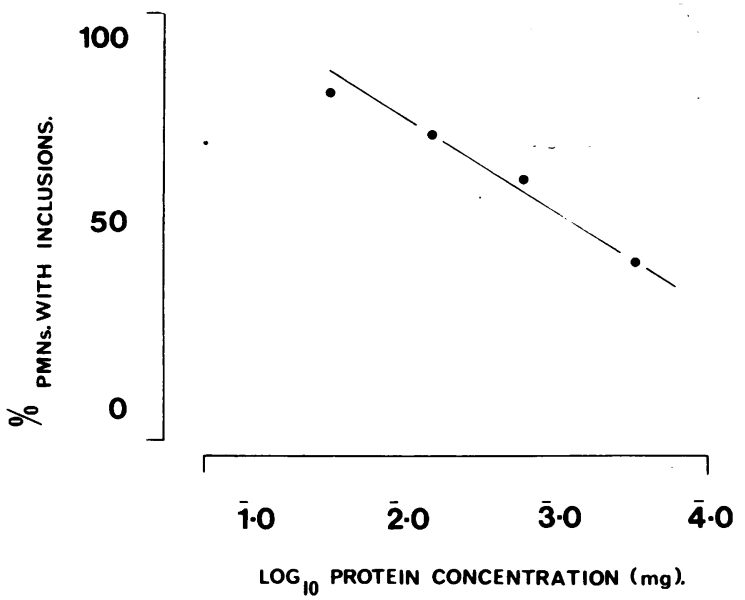

Fig. 3 Concentration: Uptake of IgG aggregates was directly proportional to $\log _{10}$ of the protein concentration over the concentration range $25-200 \mu \mathrm{g} / \mathrm{ml}^{-1}(\mathrm{n}=4$ experiments; $r=0 \cdot 724$ ).

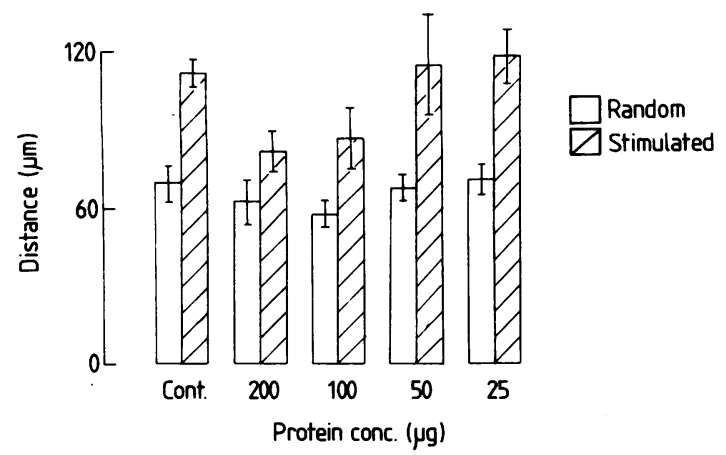

Fig. 4 Concentration: Chemotaxis only was reduced afier incubation with aggregates at a concentration of 100-200 $\mu g / m l^{-1}$. and fraction $15(>90 S)$. All fractions were of equal protein concentration $(1 \mathrm{mg} / \mathrm{gml})$, and $100 \mu \mathrm{g}$ of protein was added to $10^{6} \mathrm{PMNs}$.

Fig. 5 shows that after 30 minutes' incubation ingestion was most complete with the largest aggregates, suggesting that they are internalised most efficiently.

Aggregates of all sizes reduced PMN chemotaxis, but this effect was greatest with small aggregates $(<20 S)(\mathrm{p}<0.05$, paired $t$ test) (Fig. 6).

Prolonged incubation studies were then performed using 2 representative fractions-fraction $3(<20 S)$ and $15(>90 S)$. Double fluorochrome immunoflourescence staining confirmed that PMNs internalised small aggregates more slowly than large aggregates, and that this IF staining had largely disappeared after 360 minutes' incubation with both fractions. PMN viability remained unchanged at the end of these experiments (i.e., >98\%). When PMNs were rechallenged with IgG aggregates after 330 minutes' incubation and incubated at $37^{\circ} \mathrm{C}$ for a further 30 minutes, PMNs developed fresh immunoglobulin staining, which was both surface-bound and intracytoplasmic. Staining was predominantly

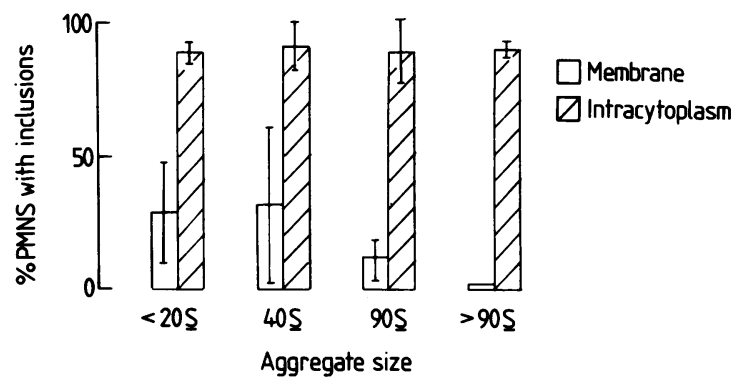

Fig. 5 Size: Double fluorochrome immunofluorescence showed that large aggregates were internalised more rapidly than small aggregates after incubation for 30 minutes.

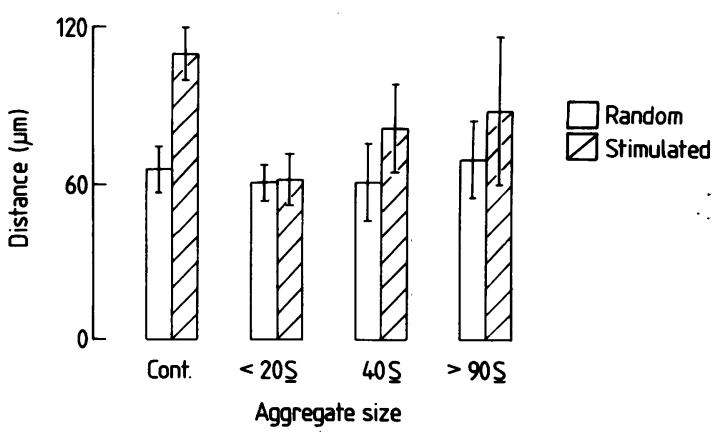

Fig. 6 Size: Aggregates of all sizes reduced PMN chemotaxis after 30 minutes' incubation, but this was maximal with small aggregates. 


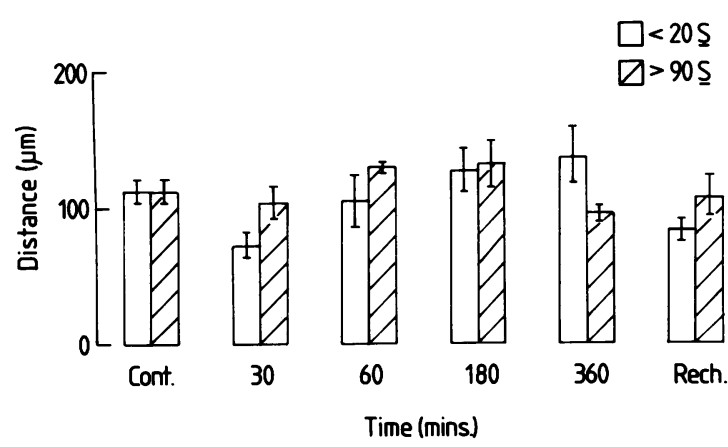

Fig. 7 Prolonged incubation studies showed that reduced and enhanced chemotaxis was a function of aggregate size.

intracytoplasmic in PMNs incubated with large aggregates and predominantly membrane-bound in PMNs incubated with small aggregates.

Fig. 7 summarises the results of migration studies performed after incubation with these 2 aggregate fractions. After 30-minutes' incubation small aggregates reduced chemotaxis only (random migration was not affected). After 60 and 180 minutes' incubation PMNs incubated with large aggregates showed enhanced chemotaxis. A similar effect was seen in PMNs incubated with small aggregates, but this occurred after a longer incubation period. When PMNs were rechallenged with IgG aggregates after 330 minutes' incubation and incubated for a further 30 minutes, only those incubated with small aggregates showed a further reduction in chemotaxis.

\section{Discussion}

These results agree with the findings of earlier work$\mathrm{ers}^{6-8}$ that IgG aggregates may stimulate as well as inhibit PMN chemotaxis. In contrast, however, Kemp and co-workers ${ }^{9}{ }^{10}$ found that IgG aggregates inhibited only random migration.

The changes observed in PMN chemotaxis were dependent on both the size and the concentration of the IgG aggregates and also on the time of incubation. PMNs incubated with whole aggregates (containing a population of aggregates of different mean sizes) first showed reduced and then enhanced chemotaxis. Further analysis of the filters showed that this increase in chemotactic activity was confined to only a small subset of PMNs, while the main population distribution remained within the first 45-60 $\mu \mathrm{m}$ of the filters.

Subsequent experiments showed that small aggregates inhibited chemotaxis and that their internalisation by PMNs took place more slowly than with large aggregates. Although both large and small aggregates enhanced PMN chemotaxis, this was seen only after a longer period of incubation with the latter. The mechanism by which aggregate uptake affects PMN migration are poorly understood. Keller et al. ${ }^{11}$ have shown that pretreating PMNs with polymerised IgG reduces their adhesion to the surface on which they are migrating. This in turn is thought to impede locomotion, since cells migrate by a continuous process of attachment and detachment to the surface on which they are moving. The presence of aggregates bound to the PMN membrane might therefore interfere with the process, resulting in impaired locomotion.

The enhanced chemotactic responsiveness we observed in a small subset of PMNs after prolonged exposure to IgG aggregates is less easily explained. Zigmond and Hirsch ${ }^{3}$ have previously shown that uptake of IgG aggregates results in the release of cell-derived chemotactic factors as well as activated complement fragments (C5a). These may stimulate neighbouring cells, but this would not explain why the effect is confined to only a few cells.

One possible explanation lies in the observation that PMNs are capable of further phagocytic activity after a refractory period. A separate receptor for chemotactically active fragments has been proposed by Kay et al. ${ }^{12}$ and they have suggested that this is situated close to the Fc receptor on the PMN membrane. Binding of $\mathrm{IgG}$ aggregates to $\mathrm{Fc}$ receptors may block binding of these chemotactically active fragments to the chemotaxis receptors. As aggregates are internalised, some unblocking of these receptors may occur, allowing further binding of chemotactically active fragments to the PMN membrane. During this period the PMN cannot bind and internalise further IgG aggregates, with the result that these chemotactic factors are unopposed and cause enhanced chemotaxis and random migration.

The need for caution in the interpretation of results is of more immediate relevance to the investigator proposing to use these techniques to study cell locomotion. Apparent changes in cell motility may be due to changes in only small subsets of PMNs, not affecting the motility of the cell population as a whole.

We are grateful to Miss E. O'Sullivan for expert secretarial assistance. Dr D. Goddard was in receipt of an Arthritis and Rheumatism Council Research Fellowship.

\section{References}

1 Brown K A, Goddard D H, Fordham J F, Currey H L F, Holborow E J. Polymorphonuclear (PMN) cell function in rheumatoid arthritis (RA) is related to surface charge. In: Preece A W, Light P A, eds. Cell electrophoresis in cancer and other clinical research. Amsterdam: Elsevier/North Holland, $1981 ; 209-16$. 
2 Mowat A G, Baum J. Chemotaxis of polymorphonuclear leucocytes from patients with rheumatoid arthritis.J Clin Invest 1971; 50: $2541-4$.

3 Zigmond S H, Hirsch J G. Leucocyte locomotion and chemotaxis. New methods for evaluation and demonstration of cell-derived chemotactic factors. J Exp Med 1973; 137: $387-410$.

4 Goddard D H, Kirk A P, Kirwan J R, Johnson G D, Holborow E J. Impaired polymorphonuclear leucocyte chemotaxis in rheumatoid arthritis. Ann Rheum Dis 1984; 43: 151-156.

5 McCarthy D, Goddard D H, Pell B K, Holborow E J. Intrinsically stable IgG aggregates. J Immunol Methods 1981; 41: 63-74.

6 Wilkinson P C. Chemotaxis and inflammation. London, Edinburgh: Churchill-Livingstone, 1975.

7 Wilkinson P C, Allen R B. Assay systems for measuring leukocyte locomotion: an overview. In: Gallin J, Quie P G, eds. Leukocyte chemotaxis. New York: Raven Press, 1978, 1-24.
8 Wilkinson P C. Effects of human IgG on locomotion of human neutrophils related to $\mathrm{IgG}$ binding of a hydrophobic probe Immunology 1980; 4: 457-66.

9 Kemp A, Roberts-Thompson P, Brown S. Inhibition of human neutrophil migration by aggregated gammaglobulin. Clin Exp Immunol 1979; 36: 334-41.

10 Kemp A, Roberts-Thompson P, Neoh S H, Brown S. Inhibition of neutrophil migration by sera from patients with rheumatoid arthritis. Clin Exp Immunol 1979; 36: 423-7.

11 Keller H U, Barandun S, Kistler P, Ploem J S. Locomotion and adhesion of neutrophil granulocytes. Effects of albumin, fibrinogen and gammaglobulins studied by reflection contrast microscopy. Exp Cell Res 1979; 122: 351-62.

12 Kay N E, Bumol T F, Douglas S D. Effect of phagocytosis and FC receptor occupancy on complement dependent neutrophil chemotaxis. J Lab Clin Med 1978; 91: 850-6.

\section{Book review}

Clinics in Rheumatic Diseases. By M. S. Jeffery and W. Carson Dick. Pp. 288. £11·75. W. B. Saunders: London. 1983.

The Clinics in Rheumatic Diseases are often a first source of reference, and their idiosyncratic choices of subjects frequently lead to unexpected and novel approaches. Although a book on laboratory tests must cover a conventional area the editors have still managed an eclectic selection with a distinctly north-eastern flavour; 12 of the 21 contributors are. from Newcastle upon Tyne. The book leads us through a whole range of laboratory approaches to rheumatology. It opens with a chapter on microbiology, passes to histopathology, haematology, immunology, and urate biochemistry, and concludes with statistics. Immunology and immunochemical measurements inevitably dominate the book, reflecting current rheumatological practice, and they account for half the chapters.

There is a clear, concise review of rheumatoid factors viewed from a historical perspective. Now that radioimmunoassays and ELISA assays for rheumatoid factors are readily available many laboratories will be trying to decide which of the available tests to use in routine clinical practice, and it is sensible to place the debate into the context of the early work of Rose and Waaler. The histopathological assessment of inflammatory synovitis remains relatively neglected compared to the immunological measures often applied, and it was pleasing to find a chapter included on this topic. Inevitably it drew attention to the pathologist's impotence in making specific diagnoses, even though this lack of specificity intrudes upon almost all laboratory tests. The short chapter on acute-phase proteins summarised present views and gave an aggressive account of the deficiencies of the current approach to investigating these proteins. The acute-phase response was suggested to be a multiple misnomer for proteins that are often associated more closely with chronic than acute inflammation; only some are phasic, and they should include peptides as well as proteins. Much research in this area was criticised as being purely phenomenological rather than concentrating on the basic scientific principles involved. Even if one disagrees with such statements they focus the subject in a vivid light. The other chapters varied but were generally good.

Most rheumatologists will find this book contains something of interest, and it will form another layer in the mound of worthwhile reading. My one disappointment was that at a time of escalating medical laboratory technology and diminishing financial resources a book on the role of the laboratory avoided the crucial question of how to limit investigations and when not to order tests. 